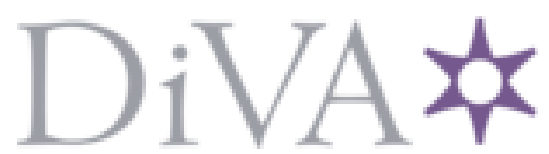

http://www.diva-portal.org

\title{
Postprint
}

This is the accepted version of a paper published in Physics Teacher. This paper has been peerreviewed but does not include the final publisher proof-corrections or journal pagination.

Citation for the original published paper (version of record):

Prytz, K. (2016)

The atomic origin of the reflection law.

Physics Teacher, 54(9): 535-537

https://doi.org/10.1119/1.4967892

Access to the published version may require subscription.

N.B. When citing this work, cite the original published paper.

Permanent link to this version:

http://urn.kb.se/resolve?urn=urn:nbn:se:mdh:diva-34232 


\section{The atomic origin of the reflection law}

Keywords: Reflection law, Ewald-Oseen theory, plane wave, Huygen's principle, dipole vibration, surface charges

\section{Introduction}

It will be demonstrated how the reflection law may be derived on an atomic basis using the plane wave approximation together with Huygen's principle. The model utilized is based on the electric dipole character of matter originating from its molecular constituents. This approach is not new but has, since it was first introduced by Ewald and Oseen in 1915 [1, 2], been applied and analysed many times before $[3,4,5,6]$. Here, we develop the Ewald-Oseen model of reflection adapted for high-school and early undergraduate students with a basic knowledge of microscopic material structure.

The reflection phenomenon is of profound importance in our daily life. Our vision, being the most important sense, rarely registers the first source of light such as the sun or a light bulb, but objects are rather visualized through reflections. Since the comprehension of nature originates from daily observations, the understanding of how vision is established is essential. Therefore, it is sensible that reflection is introduced early at school, already at around age of 10 or even earlier through discussions of images in mirrors, water surfaces and windows. At the age of 12-13 the reflection law is investigated in more detail. Based on the light ray concept, the reflection law is formulated through measurements which are repeated in more detail in high-school. As an example of a curriculum see ref. [7].

At university level first attempts to derive the reflection law from basic principles appear. In standard literature a few different approaches may be found. Some authors utilize Fermat's principle, e.g. refs. [8, 9, 10], which however is revealed as a circular reasoning since this principle is inferred from observations including reflection. A few authors involve Huygen's principle when explaining reflection, e.g. refs. $[10,11,12]$, which is a necessary but not a sufficient requirement since it doesn't specify what kind of objects are vibrating, a fact particularly problematic in case of light. In many modern textbooks this principle is not even introduced, see e.g. refs. [13, 14, 15]. Instead, the reflection and refraction laws may be derived utilizing continuity conditions appearing in electromagnetic wave theory based on Maxwell's equations, e.g. [16, 17]. This is a rather mathematically advanced method and does not promote conceptual understanding appropriate for younger students.

The Ewald-Oseen model for reflection of light may be described conceptually by the following steps:

- The source of the light ray, i.e. the plane wave, is vibrating charges.

- Light consists of an electric field oscillating with the same frequency as that of its source. 
- When light impinges on a material its charges will start to vibrate with frequency and direction dictated by the electric field. These vibrating charges will then constitute a new source of light in line with Huygen's principle.

- The type of charge is different for different material classes: solids, metals and liquids. In a dielectric solid, atoms are tightly bound to each other and have limited freedom to oscillate individually. In liquids and metals, dipolar molecules and conduction electrons respectively are individually set into oscillation by incoming light. To a good approximation, the charge oscillation may in all three cases be modelled as a dipole vibration.

- At the surface of the material a row of vibrating dipoles occur which in general vibrate with an individual phase difference appearing since the incoming plane wave will reach different points on the surface at different times. The reflection phenomena is primarily a surface effect in the optical region [10].

- The phase shift is such that the incoming and the reflected plane wave fulfil the reflection law. This will be shown below.

Following this approach the important fact that reflection should rather be considered as re-radiation is emphasized. To consider light as a substance bouncing off a surface hides many important known facts and aspects of nature.

Although light is in mind in this paper, the discussion is valid for plane waves in general.

\section{The Reflection Law}

Consider a row of oscillators having the same frequency, fig. 1 .

The direction of the plane wave generated is given by the direction of the strongest interference maxima. In the case where the oscillators have the same phase, the condition for interference maxima is given by the grating formula

$$
d \sin \gamma=n \lambda
$$

where $d$ is the distance between the oscillators, $\gamma$ is the angle between the row normal and the direction of the generated plane wave, $n$ is an integer equal to zero for the plane wave generation and $\lambda$ is the wavelength. This condition may be expressed in terms of phase

$$
\frac{2 \pi d \sin \gamma}{\lambda}=n 2 \pi
$$

In case there is a phase difference $\beta$ such that oscillator 2 is trailing, the condition for interference maxima becomes [18] 


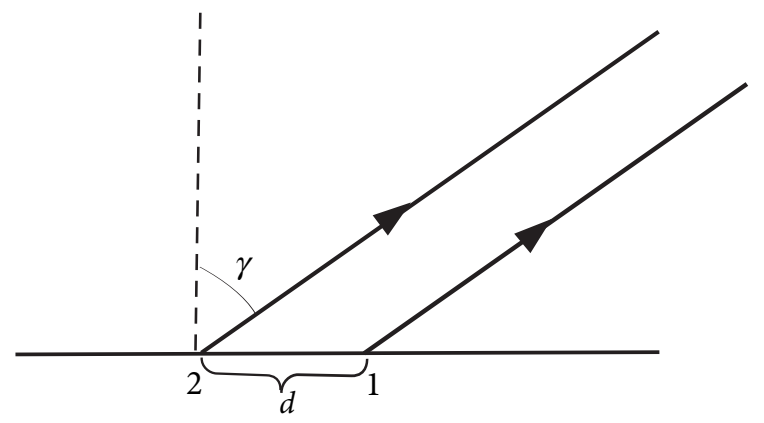

Figure 1: Plane wave radiation from a row of oscillators out of which 2 sources are indicated. The arrows indicate the rays, i.e. the direction of the plane wave propagation.

$$
\frac{2 \pi d \sin \gamma}{\lambda}-\beta=n 2 \pi
$$

According to Ewald-Oseen theory, reflection appears since the incoming wave activates the dipole oscillators at the surface. These absorb the energy and start to oscillate. Thereby they form a source to a new wave which in one direction is comprehended as the reflected wave and in the opposite direction as the refracted wave. In case of oblique incidence the incoming wave activates the oscillators one after another, fig. 2.

Therefore, at the surface a row of oscillators occurs vibrating out of phase, where two neighbours have a constant phase difference $\beta$ given by

$$
\beta=\omega \Delta t
$$

Here $\omega$ is the angular velocity of the vibrators which equal that of the wave. From fig. 2 it is seen that the time lapse between activation of two neighbouring oscillators is given by

$$
\Delta t=\frac{d \sin \alpha}{c}
$$

where $c$ is the speed of wave propagation and $d$ is the distance between two neighbouring oscillators. Introducing the two last formulas in formula (3) the interference maximum condition becomes

$$
\frac{2 \pi d \sin \gamma}{\lambda}-\omega \frac{d \sin \alpha}{c}=n 2 \pi
$$

Since $\frac{\omega}{c}=\frac{2 \pi}{\lambda}$, the condition may be written 


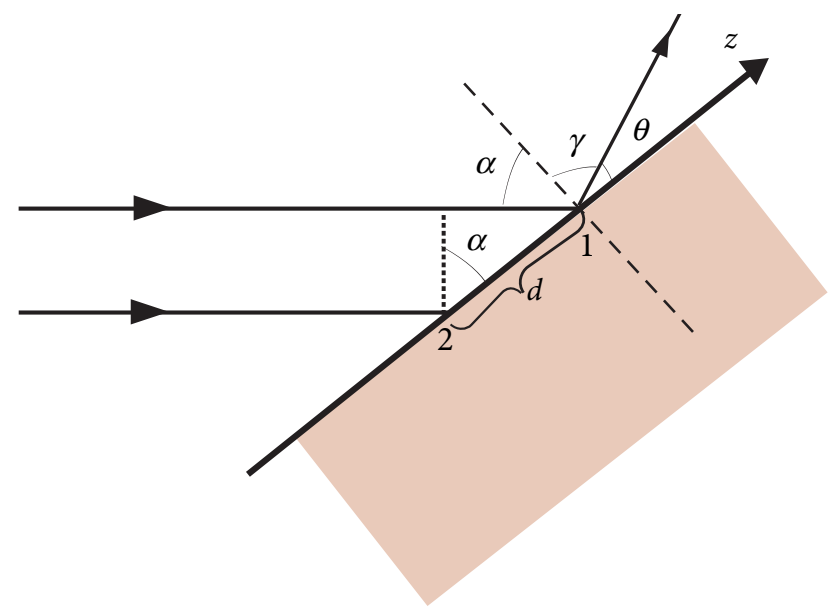

Figure 2: A plane wave is incident on an intersection. Two oscillators at the surface are indicated, numbered 1 and 2 .

$$
\frac{2 \pi d \sin \gamma}{\lambda}-\frac{2 \pi d \sin \alpha}{\lambda}=n 2 \pi
$$

Setting $n=0$, it is obtained

$$
\sin \gamma=\sin \alpha
$$

which is the reflection law.

Thus, this law is far from trivial since it reveals the inner structure of matter. It holds for all waves and all kind of matter as long as the distance $d$ between two neighbouring oscillators is smaller than wavelength $\lambda$, else higher-order reflections may occur $(n>0)$ indicating a break-down of the simple plane wave approximation.

\section{Discussion}

The reflection phenomena provides a possibility to develop the students' knowledge of the microscopic dynamics of nature. Having this knowledge, several other phenomena may be understood at the same level. Some examples are:

The refraction law which may be derived in the same manner with the sole alteration being the wave speed in the second material.

Brewster reflection appearing when the dipoles at the surface align in the re-radiation direction.

The material dependence of speed of light governed by the refraction index [19]. 
It is also noteworthy that the above theory of reflection is the same adopted when dealing with directed antenna arrays, so called phased arrays [4]. Here, a desired radiation direction may be chosen by electronically designing a proper phase shift between the antenna elements, both for sending and receiving. Therefore, within the proposed approach to reflection, students also gain insight into fundamentals of antenna theory with applications in for example communication, surveying and astronomy.

\section{References}

[1] PP Ewald, Ann. Phys. 49 (1916) 1

[2] CW Oseen, Ann. Phys. 48 (1915) 1

[3] E Lalor, E Wolf, J. Opt. Soc. A. 62 (1972) 1165

[4] H Fearn, DFV James, PW Milloi, Am. J. Phys. 64 (1996) 986

[5] F Carreno, M Anton, Eur. J. Phys. 20 (1999) 443

[6] W Shu et al., arXiv:physics/0609224v2 [physics.optics]

[7] Nasa Educational Product, 'Optics: Light, color and their uses', www.nasa.gov/pdf/58258main Optics.Guide.pdf

[8] RP Feynman, RB Leighton, ML Sands, 'The Feynman Lectures on Physics', Volume 1, Addison-Wesley, Reading, Massachusetts, 1989

[9] A Montwill, A Breslin, 'Let there be Light', Imperial College Press, London, 2013

[10] E Hecht, 'Optics', Pearson, 2013

[11] H Benson, 'University Physics', John Wiley and Sons, 1996

[12] J Touger, 'Introductory Physics: Building Understanding', John Wiley and Sons, 2006

[13] PS Hewitt, J Suchroki, LA Hewitt, 'Conceptual Physical Science', Addison Wesley Lngman, 1999

[14] S Holzner, 'Physics for Dummies', Wiley, Indianapolis, 2006

[15] CH Holbrow, JN Lloyd, JL Amato, 'Modern Introductory Physics', Springer, New York, 1999

[16] JD Jackson, 'Classical Electrodynamics', Wiley, 1999

[17] RK Wangsness,'Electromagnetic Field', Wiley, 1986

[18] N Yu et al, Science 334 (2011) 333

[19] MB James, DJ Griffiths, Am. J. Phys. 60 (4) 1992309 